\title{
Splicing mutation of a gene within the Duchenne muscular dystrophy family
}

\author{
Y.B. Zhu ${ }^{1,2 *}$, J.H. Gan ${ }^{3 *}$, J.W. Luo ${ }^{1,2}$, X.Y. Zheng ${ }^{1,2}$, S.C. Wei ${ }^{2}$ and D. $\mathrm{Hu}^{2}$ \\ ${ }^{1}$ Provincial Medical College, Fujian Medical University, \\ Fujian University of Traditional Chinese Medicine, Fuzhou, China \\ ${ }^{2}$ Department of Traditional Chinese Medicine, Fujian Provincial Hospital, \\ Fuzhou, China \\ ${ }^{3}$ School of Clinical Medicine, Capital Medical University, Beijing, China \\ *These authors contributed equally to this study. \\ Corresponding author: J.W. Luo \\ E-mail: docluo@126.com
}

Genet. Mol. Res. 15 (2): gmr.15018258

Received December 11, 2015

Accepted February 2, 2016

Published July 15, 2016

DOI http://dx.doi.org/10.4238/gmr.15028258

\begin{abstract}
The aim of this study was to identify the mutation site and phenotype of the Duchenne muscular dystrophy $(D M D)$ gene in a DMD family. The $D M D$ gene is by far the largest known gene in humans. Up to $34 \%$ of the point mutations reported to date affect splice sites of the $D M D$ gene. However, no hotspot mutation has been reported. Capture sequencing of second-generation exons was used to investigate the $D M D$ gene in a proband. Sanger sequencing was performed for mutation scanning in eight family members. Scale-invariant feature transform and PolyPhen were applied to predict the functional impact of protein mutations. A hemizygous splicing mutation IVS44ds $+1 \mathrm{G}-\mathrm{A}$ (c. $6438+1 \mathrm{G}>\mathrm{A})$ that induces abnormal splicing variants during late transcription and produces abnormal proteins was located in intron 44. Four missense mutations (p.Arg2937Gln, p.Asp882Gly, p.Lys2366Gln, and p.Arg1745His) that are known multiple-polymorphic sites were found in the coding region of the $D M D$ gene. A heterozygous c.6438
\end{abstract}


$+1 \mathrm{G}>\mathrm{A}$ mutation was detected on the $\mathrm{X}$ chromosome of the proband's mother and maternal grandmother.

Key words: Capture sequencing of second-generation exons; Duchenne muscular dystrophy; Gene; Mutation; Pedigree study

\section{INTRODUCTION}

Pseudo-hypertrophic muscular dystrophy (PHMD), first described by Duchenne et al. in 1868, is an inherited and lethal X-linked recessive neuromuscular disorder. PHMD can be divided into two distinct types: Duchenne muscular dystrophy (DMD, OMIM310200), and Becker muscular dystrophy (BMD, OMIM300376). In 1953, Becker initially described BMD as a benign X-linked variant of DMD. DMD and BMD are caused by mutations of the dystrophin gene (also known as the $D M D$ gene) on the $\mathrm{X}$ chromosome at position Xp21.2. Three different types of mutation exist: 1) a partial gene deletion (55-65\%); 2) a gene duplication (5-15\%); and 3) a point mutation (35\%). Approximately $70 \%$ of PHMD diagnoses result from carrier mothers and $30 \%$ of these cases occur as a result of spontaneous mutation (Aartsma-Rus et al., 2006; Flanigan et al., 2009; Takeshima et al., 2010). Both DMD and BMD are characterized by progressive skeletal muscle weakness, muscular dystrophy, Gower's sign, possible intellectual disability, or cardiomyopathy. The progressive muscle weakness of DMD usually starts by age 5 . The ability to walk may be lost by age 12 . Young patients with DMD typically live into their twenties; however, they typically have breathing difficulties and heart disease. BMD is less severe than DMD; with BMD, the ability to walk may be lost after age 16 .

PHMD (including DMD and BMD) is an inherited and lethal X-linked recessive neuromuscular disorder. DMD affects approximately 1 in 3500 male births worldwide (Hoffman et al., 1988). Meta-analysis of international prevalence revealed that the pooled prevalence values for DMD and BMD were $4.78(95 \% \mathrm{CI}=1.94-11.81)$ and $1.53(95 \% \mathrm{CI}$ $=0.26-8.94)$ per 100,000 males, respectively. The incidence of DMD ranged from 10.71 to 27.78 per 100,000 males (Mah et al., 2014). Although theoretically DMD does not occur in women, both male and female DMD patients have been reported in clinical practice; this might be caused by a secondary mutation or failure in epigenetic regulation in female carriers (Yoon et al., 2011; Brioschi et al., 2012). Therefore, this occurrence in women highlights the importance of conducting genetic testing for differential diagnoses between autosomal recessive limb-girdle muscular dystrophy and DMD. The goal of the present study was to determine the mutation site and phenotype of the $D M D$ gene in a Chinese family.

\section{MATERIAL AND METHODS}

\section{Patients}

One male patient diagnosed with DMD at the First Affiliated Hospital of Fujian Medical University (also known as Fujian Provincial Hospital) and eight members of his family were included in this study (Figure 1). The study was approved by the Medical Ethics Committee of Fujian Provincial Hospital in Fuzhou, Fujian, China. Written informed consent was obtained from all participants and guardians of minors. 


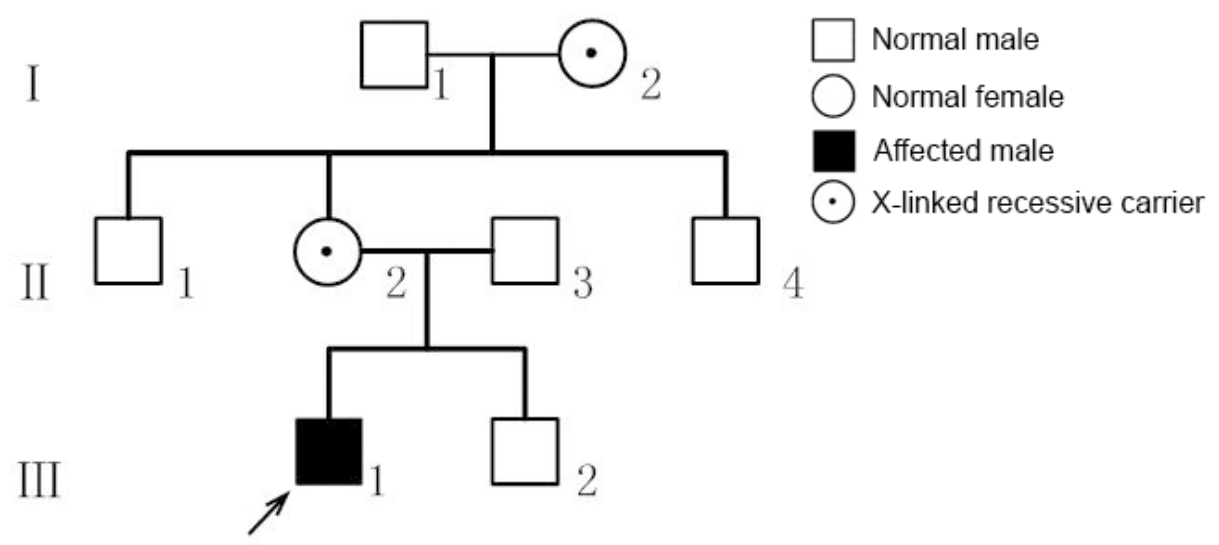

Figure 1. Pedigree of a family with Duchenne muscular dystrophy $(\mathrm{DMD})$ genes. $\mathrm{I}=1$ st generation, $\mathrm{II}=2 \mathrm{nd}$ generation, III = 3rd generation, arrow = proband .

\section{Clinical studies}

All the family members included in the study were evaluated by cardiac ultrasound examination, X-ray investigation, computed tomography, electrocardiography, and blood, urine, and biochemical tests. Electromyography and magnetic resonance imaging of muscles were carried out on the proband.

\section{Genomic DNA extraction}

DNA samples from the proband ( $15 \mathrm{~mL}$ peripheral blood) and seven family members ( $2 \mathrm{~mL}$ peripheral blood) were extracted using standard techniques with an Omega EZNA ${ }^{\circledR}$ Tissue DNA Kit, (Omega Bio-Tek, Doraville, GA, USA).

The proband's DNA sample was analyzed by Illumina sequencing (He et al., 2011; Wu et al., 2011), and bioinformatic analysis (Li et al., 2008) was carried out using a Nanodrop 2000

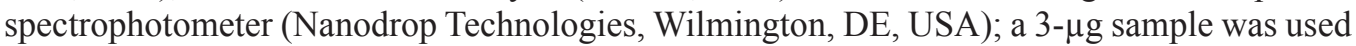
for DNA fragmentation analysis. The DNA was cut using the Covaris method and produced 250-bp DNA fragments. Next, an end repair reaction was performed and end repaired products were linked with a screening-specific adapter. The amplification reaction was performed according to the general adapter primer binding sites with further purification. Target gene enrichment was carried out using a DNA capture chip for sequencing by the Illumina 2000 sequencer (Hiseq2000, Macrogen, Seoul, Republic of Korea). Original images were analyzed using the Illumina base-calling Software 1.7 to collect 90-bp reads. Sequencing analysis was performed after deletion of poorly qualified reads and adapter sequences. The simple object access protocol (SOAP) software was used to analyze the copy number, polymorphism and insertion/deletion, and screening for genes suspected of being pathogenic. SIFT (http:// sift.jcvi.org/) and PolyPhen softwares (http://genetics.bwh.harvard.edu/pph/) were used for protein function prediction. These procedures were carried out in the Shenzhen Genomics Research Institute, Shenzhen, Guangdong, China. 


\section{Sanger sequencing}

$D M D$ gene sequences were obtained from GenBank (NM_004006). The Primer Premier 5 software was used to design exons $44-45$ of the $D M D$ gene. Primers were synthesized by Beijing Liuhe Hua Genetic Technology Co., Ltd., Beijing, China, including F: 5'-CTAACAGAAGCTGAACAGTTTCTCAG-3', and R: 5'-TGTTACGATGCTTCCCTCTGTCACAG-3'. Polymerase chain reaction (PCR) was carried out in a reaction volume of $25 \mu \mathrm{L}$ containing $10 \mathrm{X}$ Ex Taq buffer $2.5 \mu \mathrm{L}$, dNTP $(2.5 \mathrm{mM}) 2 \mu \mathrm{L}$, forward primer $(3 \mathrm{mM}) 3 \mu \mathrm{L}$, reverse primer $(3 \mathrm{mM}) 3 \mu \mathrm{L}$, DNA template $1 \mu \mathrm{L}$, Ex Taq $0.2 \mu \mathrm{L}$, $\mathrm{H}_{2} \mathrm{O} 18.3 \mu \mathrm{L}$ for further amplification using PTC-200 PCR (BIO-RAD, Hercules, CA, USA). After a 5-min denaturation at $94^{\circ} \mathrm{C}, \mathrm{PCR}$ amplification was carried out using the following cycle profile: denaturation at $94^{\circ} \mathrm{C}$ for $40 \mathrm{~s} ; 35$ cycles of annealing at $57^{\circ} \mathrm{C}$ for $40 \mathrm{~s}$ and extension at $72^{\circ} \mathrm{C}$ for $60 \mathrm{~s}$; and extension at $72^{\circ} \mathrm{C}$ for 10 min to produce 359 -bp PCR products. An EZNA Gel Extraction Kit (Omega Company) was used to purify the PCR products. Collected PCR products were directly used for a sequencing reaction according to standard protocols (BigDye Terminator v1.1 Kit, Applied Biosystems, Foster City, CA, USA). Comparison of the analyzed sequence with that of a normal sequence was performed using DNAman Version 5.2.2 software (Lynnon Biosoft, Quebec, Canada). The PCR products were then cloned into the pGEM $^{\circledR}$-T Easy (Promega, Madison, WI, USA) vector to detect heterozygous deletions or insertions.

\section{RESULTS}

\section{Demographic and clinical studies}

A 12-year-old male presented progressive bilateral lower extremity weakness and soreness evolving over 2 years. He could walk, but his ability to squat, stand up, and climb stairs was limited. Physical examination revealed intellectual impairment, mild dystrophy of the bilateral proximal lower extremities, and enlargement of the calf muscles. In the lower extremities, the patient's strength was $3+/ 5$ proximally and 5-/5 distally. Abnormal laboratory findings included creatine kinase (11,930 IU/L), CK-MB isoenzyme (320 IU/L), aspartate aminotransferase (253 $\mathrm{IU} / \mathrm{L})$, alanine aminotransferase (299 IU/L), lactate dehydrogenase $(827 \mathrm{IU} / \mathrm{L})$, serum uric acid $(622 \mu \mathrm{M})$, serum creatinine $(13 \mu \mathrm{M})$, troponin I $(0.28 \mu \mathrm{g} / \mathrm{L})$ and erythrocyte sedimentation rate $(2.0 \mathrm{~mm} / \mathrm{h})$. Electrocardiography indicated sinus tachycardia with a heart rate of $140 \mathrm{bpm}$ and abnormal Q waves (I, aVL, V3-V6). The electromyography results showed myopathic changes. The pulmonary computed tomography and cardiac sonography examinations were negative. Previous multiplex ligation-dependent probe amplification reported absence of DMD DNA copy number variations. In the muscular magnetic resonance imaging, several muscles including obturatorius, sartorius, gluteus maximus, rectus femoris, semimembranosus, semitendinosus, lateral, adductor, biceps femoris, and thigh muscles showed different degrees of atrophy, and the gracilis muscle had mild hypertrophy. The presence of subcutaneous fat increased significantly with fascia connective tissue hyperplasia and hypertrophy.

\section{Sequence capture}

Figure 2 shows the results of sequence capture of the $D M D$ exon for the proband. The coverage ratio for exon 79 was over $99 \%$. The mean and median depth had similar values demonstrating random sequencing. 


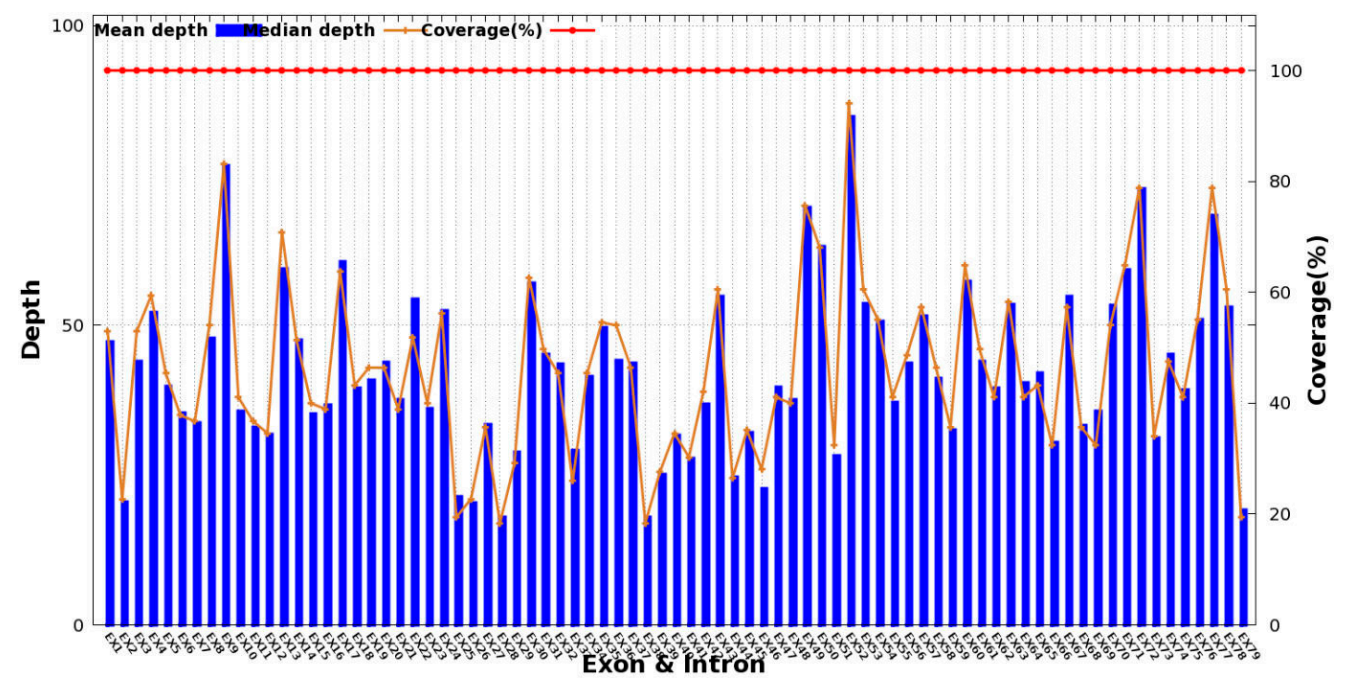

Figure 2. Exon sequencing capture array of the Duchenne muscular dystrophy (DMD) gene. Mean depth (X): average depths at each base in the exon; median depth $(\mathrm{X})$ : the depth separating the higher half of all depths; coverage (\%): the percentage of bases in all targeted regions.

\section{Mutation analysis}

In individual III 1 (proband), DMD exon sequencing analysis revealed no large deletions or duplications of the $D M D$ gene. A hemizygous splicing mutation IVS44ds $+1 \mathrm{G}$ A (c.6438 $+1 \mathrm{G}>\mathrm{A})$, located in intron 44 , created abnormal splicing variants during late transcription, and these produced abnormal proteins. This might represent a potentially pathogenic mutation because it rarely occurs in a normal population (Figure 3). Four missense mutations (p.Arg2937Gln, p.Asp882Gly, p.Lys2366Gln, and p.Arg1745His) that are known multiple-polymorphic sites were found in the coding region of the $D M D$ gene.

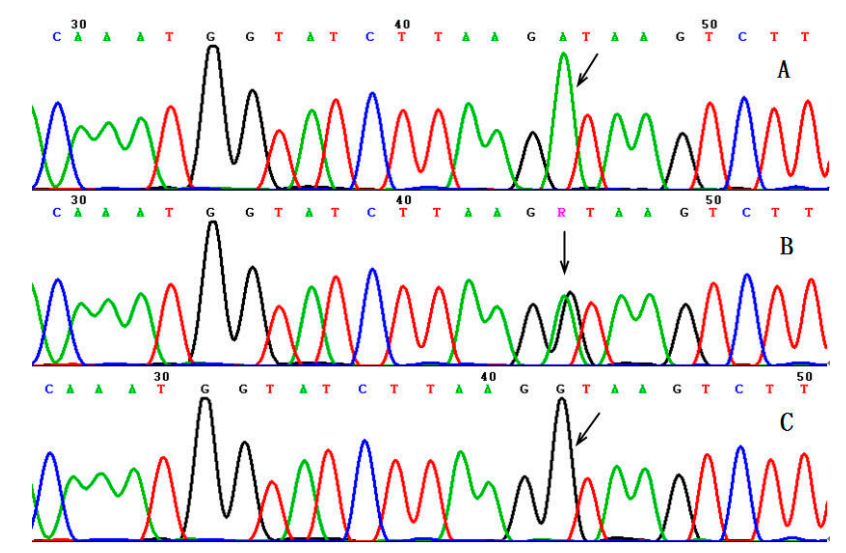

Figure 3. Sequencing of the region between exon 44 and intron 44 splicing site of the Duchenne muscular dystrophy $(D M D)$ gene in the DMD family. A. A hemizygous splicing mutation IVS44ds $+1 \mathrm{G}-\mathrm{A}$ (c. $6438+1 \mathrm{G}>\mathrm{A}$, $\mathrm{X}$ chromosome) of the proband's $D M D$ gene. B. A heterozygous c. $6438+1 \mathrm{G}>\mathrm{A}$ was carried on the $D M D$ gene of the proband's mother. C. Normal sequencing of the proband's father and brother. 
Sanger sequencing in the eight family members reported that the hemizygous mutation IVS44ds $+1 \mathrm{G}-\mathrm{A}$ (c.6438 $+1 \mathrm{G}>\mathrm{A}$ ) was found on the $\mathrm{X}$ chromosome in individual III 1. A heterozygous c. $6438+1 \mathrm{G}>\mathrm{A}$ mutation was carried on the $\mathrm{X}$ chromosome of individuals I 2 and 2 but not on that of individuals I 1, II 1, 3, 4, and III 2 (Table 1). These finding raised the possibility of a splicing mutation, IVS44ds $+1 \mathrm{G}-\mathrm{A}(\mathrm{c} .6438+1 \mathrm{G}>\mathrm{A})$, in the region between the exon 44 and intron 44 splicing site of the DMD gene as causative mutations in the DMD family.

\begin{tabular}{|c|c|c|c|c|c|c|c|c|}
\hline Mutation & RS-ID & Nucleotide change & Position & Predicted effect & Frequency in dbSNP & Frequency in HapMap & Frequency in 1000 Genomes Project & Frequency in BGI \\
\hline \multicolumn{9}{|c|}{$D M D$ gene (NM_004006) } \\
\hline p.Arg2937Gln & rs1800280 & c. $8810 \mathrm{~A}>\mathrm{G}$ & Exon 59 & Missense & 0.877 & 0.908 & 0.4588 & 0.9024 \\
\hline p.Lys2366Gln & rs 1800275 & c. $7096 \mathrm{C}>\mathrm{A}$ & Exon 48 & Missense & 0.041 & 0.044 & 0.0266 & 0.0802 \\
\hline IVS44ds+1G-A & & c. $6438+1 \mathrm{G}>\mathrm{A}$ & Intron 44 & Silent & 0 & 0 & 0 & 0 \\
\hline p.Arg1745His & \begin{tabular}{|l|l|} 
rs1801187 \\
\end{tabular} & c. $5234 \mathrm{G}>\mathrm{A}$ & Exon 37 & Missense & 0.201 & 0.622 & 0.1429 & 0.6301 \\
\hline p.Asp882Gly & rs 228406 & c. $2645 \mathrm{G}>\mathrm{A}$ & Exon 21 & Missense & 0.533 & 0.847 & 0.2793 & 0.8615 \\
\hline
\end{tabular}

Frequency information about the single nucleotide polymorphisms (SNPs) is included in the dbSNP database. Frequency information about the Asian population SNPs is included in the HapMap database. Frequency information about the SNPs from total sequencing samples is included in the 1000 Genomes Project. Frequency information about the SNPs from $\sim 625$ normal sequencing samples is from the Beijing Genomics Institute (BGI).

\section{DISCUSSION}

The $D M D$ gene was initially located on the short arm of the $\mathrm{X}$ chromosome (Xp21) by Lindenbaum et al. in 1979. In 1983, Kingston et al. found that the $B M D$ gene had the chromosomal locus Xp21. Brown et al. (1985) revealed that linkage analysis of the DNA polymorphisms in relation to the two disorders showed similar genetic distances. Koenig et al. (1987) used molecular cloning of full-length complementary DNA (cDNA) to confirm that DMD and BMD had different mutations in the $D M D$ gene. The $D M D$ gene is by far the largest known gene in humans, spanning $2400 \mathrm{~kb}$ in 79 exons and 78 introns. This gene comprises almost $0.1 \%$ of the human genome or about $1.5 \%$ of the entire X chromosome. cDNA sequences that code for the protein dystrophin account for just $1 \%$ of the genome. Dystrophinopathy is characterized by a spectrum of muscle diseases caused by DMD mutation-induced protein dystrophin deficiency. Four clinical types of dystrophinopathy exist: DMD, BMD (including asymptomatic high creatine kinase hyperlipidemia, muscle cramps and myalgia, quadriceps myopathy), X-linked dilated cardiomyopathy, and asymptomatic or symptomatic carriers.

A search of the human genome database (HGMD Professional 2014.4) found that at least 2982 mutations occur in the $D M D$ gene resulting in a DMD genotype that may include 589 missense or nonsense mutations, 257 splicing mutations, 1 regulatory mutation, 347 small deletions, 122 small insertions, 34 small insertions and deletions, 1054 gross deletions, 493 gross insertions, or 85 complex rearrangements. No mutation hotspot has been reported. Despite the challenges caused by the length of the $D M D$ gene and its various mutations, few novel methods exist that would benefit the detection of mutations in the $D M D$ gene, e.g., multiplex ligation-dependent probe amplification ( $\mathrm{Li}$ et al., 2013). Li et al. (2011) studied 720 DMD cases in China and reported a detection rate of $64.9 \%$ (54.3\% for gene deletions, $10.6 \%$ for gene duplications). The deletion mutation rate in exon $45-54$ was $71.9 \%(281 / 391)$ in patients with gene deletions. Gene duplications in exon 1-40 occurred in $82.9 \%(63 / 76)$ of all gene duplications. In all gene deletion and duplication cases, the rate of DMD and intermediate muscular dystrophy was $90.6 \%$ (423/467), and that of BMD was $9.4 \%$ (44/467). 
These results indicate that DMD is mainly caused by dystrophin gene deletion mutations. Mutations were not uniformly distributed in mutation hotspots, as would be expected. Secondgeneration exons capture sequencing demonstrates a high detection rate of DMD mutations.

Recent studies have shown that no correlation exists between deletion and/or duplication size of the $D M D$ gene and clinical phenotypes and disease severity (Malhotra et al., 1988; Li et al., 2013). Microdeletions in exon 44 led to typical DMD, while gross deletions (approximately 50\%) resulted in BMD (Love et al., 1991). Deletions in the central portion and distal rod-like region caused muscle cramps and myalgia, and some other mutations caused asymptomatic high creatine kinase hyperlipidemia (Melis et al., 1998). This was explained by the reading frame hypothesis (Monaco et al., 1988), which states that mutations that disrupt the reading frame (out-of-frame/frame-shift) produce shorter abnormal proteins that lack the main function region (a cysteine-rich C-terminal region), and usually lead to dystrophin deficiency, eventually causing DMD. In BMD patients, however, mutations maintained the reading frame (in-frame mutations), and generally resulted in abnormal but partly functional dystrophin. The reading frame rule explained more than $90 \%$ of DMD and BMD cases (Zhou et al., 2006).

Splicing mutations are common and account for $15 \%$ of mutations in humans (Cooper and Mattox, 1997). Up to 34\% of the point mutations reported to date affect splicing sites of the DMD gene (Tuffery-Giraud et al., 1999). To the best of our knowledge, the rare splicing sites that we found in this study were similar to those found in a pedigree research study by Flanigan et al. (2009). The splicing mutation might change an RNA precursor splicing mechanism leading to abnormal splicing variants during late transcription and abnormal proteins. Recently, it has been demonstrated that mutations of pseudoexons within introns may cause activation of pseudoexons or cryptic exons (Béroud et al., 2004). A single-site mutation may activate potential splicing sites that transform introns into exons, usually leading to extra insertions into mature mRNA forming a termination codon. Translation termination occurs early when reading reaches a termination codon and eventually leads to DMD.

\section{CONCLUSION}

In summary, our findings suggest that the splicing mutation IVS44ds $+1 \mathrm{G}-\mathrm{A}$ (c.6438 $+1 \mathrm{G}>\mathrm{A}$ ) in the $D M D$ gene might be a potentially pathogenic mutation in the DMD family. To the best of our knowledge, these rare splice sites were found for the second time in the present study.

\section{Conflicts of interest}

The authors declare no conflict of interest.

\section{ACKNOWLEDGMENTS}

We thank the Beijing Genomics Institute (BGI) for performing second-generation DNA sequencing for this research project. Research supported by the Science and Technology Project of Fujian Province (Grant \#2014Y0007), the Financial Scheme for the Young Talent Training Program of Fujian Health Industry (Grant \#2015-ZQN-ZD-7), and the Fujian Province Traditional Chinese Medicine Research Project (Grant \#wzsb201317). 


\section{REFERENCES}

Aartsma-Rus A, Van Deutekom JC, Fokkema IF, Van Ommen GJ, et al. (2006). Entries in the Leiden Duchenne muscular dystrophy mutation database: an overview of mutation types and paradoxical cases that confirm the reading-frame rule. Muscle Nerve 34: 135-144. http://dx.doi.org/10.1002/mus.20586

Béroud C, Carrié A, Beldjord C, Deburgrave N, et al. (2004). Dystrophinopathy caused by mid-intronic substitutions activating cryptic exons in the DMD gene. Neuromuscul. Disord. 14: 10-18. http://dx.doi.org/10.1016/S09608966(03)00169-X

Brioschi S, Gualandi F, Scotton C, Armaroli A, et al. (2012). Genetic characterization in symptomatic female DMD carriers: lack of relationship between X-inactivation, transcriptional DMD allele balancing and phenotype. $B M C$ Med. Genet. 13: 73. http://dx.doi.org/10.1186/1471-2350-13-73

Brown CS, Thomas NS, Sarfarazi M, Davies KE, et al. (1985). Genetic linkage relationships of seven DNA probes with Duchenne and Becker muscular dystrophy. Hum. Genet. 71: 62-74. http://dx.doi.org/10.1007/BF00295671

Cooper TA and Mattox W (1997). The regulation of splice-site selection, and its role in human disease. Am. J. Hum. Genet. 61: 259-266. http://dx.doi.org/10.1086/514856

Flanigan KM, Dunn DM, von Niederhausern A, Soltanzadeh P, et al.; United Dystrophinopathy Project Consortium (2009). Mutational spectrum of DMD mutations in dystrophinopathy patients: application of modern diagnostic techniques to a large cohort. Hum. Mutat. 30: 1657-1666. http://dx.doi.org/10.1002/humu.21114

He J, Wu J, Jiao Y, Wagner-Johnston N, et al. (2011). IgH gene rearrangements as plasma biomarkers in Non- Hodgkin's lymphoma patients. Oncotarget 2: 178-185. http://dx.doi.org/10.18632/oncotarget.235

Hoffman EP, Fischbeck KH, Brown RH, Johnson M, et al. (1988). Characterization of dystrophin in muscle-biopsy specimens from patients with Duchenne's or Becker's muscular dystrophy. N. Engl. J. Med. 318: 1363-1368. http:// dx.doi.org/10.1056/NEJM198805263182104

Kingston HM, Harper PS, Pearson PL, Davies KE, et al. (1983). Localisation of gene for Becker muscular dystrophy. Lancet 2: 1200. http://dx.doi.org/10.1016/S0140-6736(83)91252-7

Koenig M, Hoffman EP, Bertelson CJ, Monaco AP, et al. (1987). Complete cloning of the Duchenne muscular dystrophy (DMD) cDNA and preliminary genomic organization of the DMD gene in normal and affected individuals. Cell 50: 509-517. http://dx.doi.org/10.1016/0092-8674(87)90504-6

Li Q, Li SY, Zhang HM, He WZ, et al. (2013). Clinical value of MLPA in the prenatal gene diagnosis of Duchenne muscular dystrophy. Zhonghua Fu Chan Ke Za Zhi 48: 161-164.

Li R, Li Y, Kristiansen K and Wang J (2008). SOAP: short oligonucleotide alignment program. Bioinformatics 24: 713714. http://dx.doi.org/10.1093/bioinformatics/btn025

Li SY, Sun XF, Li Q, Zhang HM, et al. (2011). [Association of mutation types and distribution characteristics of dystrophin gene with clinical symptoms in Chinese population]. Yi Chuan 33: 251-254. http://dx.doi.org/10.3724/ SP.J.1005.2011.00251

Lindenbaum RH, Clarke G, Patel C, Moncrieff M, et al. (1979). Muscular dystrophy in an X; 1 translocation female suggests that Duchenne locus is on X chromosome short arm. J. Med. Genet. 16: 389-392. http://dx.doi.org/10.1136/ jmg.16.5.389

Love DR, Flint TJ, Genet SA, Middleton-Price HR, et al. (1991). Becker muscular dystrophy patient with a large intragenic dystrophin deletion: implications for functional minigenes and gene therapy. J. Med. Genet. 28: 860-864. http:// dx.doi.org/10.1136/jmg.28.12.860

Mah JK, Korngut L, Dykeman J, Day L, et al. (2014). A systematic review and meta-analysis on the epidemiology of Duchenne and Becker muscular dystrophy. Neuromuscul. Disord. 24: 482-491. http://dx.doi.org/10.1016/j. $\underline{\text { nmd.2014.03.008 }}$

Malhotra SB, Hart KA, Klamut HJ, Thomas NS, et al. (1988). Frame-shift deletions in patients with Duchenne and Becker muscular dystrophy. Science 242: 755-759. http://dx.doi.org/10.1126/science.3055295

Melis MA, Cau M, Muntoni F, Mateddu A, et al. (1998). Elevation of serum creatine kinase as the only manifestation of an intragenic deletion of the dystrophin gene in three unrelated families. Eur. J. Paediatr. Neurol. 2: 255-261. http:// dx.doi.org/10.1016/S1090-3798(98)80039-1

Monaco AP, Bertelson CJ, Liechti-Gallati S, Moser H, et al. (1988). An explanation for the phenotypic differences between patients bearing partial deletions of the DMD locus. Genomics 2: 90-95. http://dx.doi.org/10.1016/0888$\underline{7543(88) 90113-9}$

Takeshima Y, Yagi M, Okizuka Y, Awano H, et al. (2010). Mutation spectrum of the dystrophin gene in 442 Duchenne/ Becker muscular dystrophy cases from one Japanese referral center. J. Hum. Genet. 55: 379-388. http://dx.doi. org/10.1038/jhg.2010.49 
Tuffery-Giraud S, Chambert S, Demaille J and Claustres M (1999). Point mutations in the dystrophin gene: evidence for frequent use of cryptic splice sites as a result of splicing defects. Hum. Mutat. 14: 359-368. http://dx.doi.org/10.1002/ (SICI) 1098-1004(199911)14:5<359::AID-HUMU1>3.0.CO;2-K

Wu J, Matthaei H, Maitra A, Dal Molin M, et al. (2011). Recurrent GNAS mutations define an unexpected pathway for pancreatic cyst development. Sci. Transl. Med. 3: 92ra66. http://dx.doi.org/10.1126/scitranslmed.3002543

Yoon J, Kim SH, Ki CS, Kwon MJ, et al. (2011). Carrier woman of Duchenne muscular dystrophy mimicking inflammatory myositis. J. Korean Med. Sci. 26: 587-591. http://dx.doi.org/10.3346/jkms.2011.26.4.587

Zhou GQ, Xie HQ, Zhang SZ and Yang ZM (2006). Current understanding of dystrophin-related muscular dystrophy and therapeutic challenges ahead. Chin. Med. J. (Engl.) 119: 1381-1391. 